\title{
A SYSTEMATIC STUDY OF IRAS SELECTED PROTO-PLANETARY NEBULA CANDIDATES
}

\author{
J.Y. HU and B.W. JIANG \\ Beijing Observatory, Academia Sinica, Beijing, 100080, China \\ and \\ T. DE JONG and S. SLIJKHUIS \\ Astronomical Institute, University of Amsterdam, The Netherlands
}

A complete sample of PPN candidates was selected mainly based on the IRAS colors(Hu et al.,1990). For total 62 unknown objects in the sample we have made 1. ground-based infrared astrometry; 2. optical identifications; 3 . near infrared and optical photometry; 4 . optical spectroscopic observations; 5 . radio molecular line observations $(\mathrm{OH}$ maser and $\mathrm{CO}$ thermal lines); and 6 . check the optical variations from historical plates. Some additional observations such as high resolution spectraoscopic, optical/NIR CCD imaging observations for particularly interested objects were also carried out.

We conclude that the nature of most objects in the sample is PPN on the following grounds.

1. 20 out of 21 objects with sufficiently high $\mathrm{S} / \mathrm{N}$ in their spectra for a reliable luminosity classification, appear indeed to be supergiant-like(but IRAS16552-3050 is a giant).

2. The average luminosities are $3000 \mathrm{Lo}$ for 10 confirmed carbon-rich objects and 7300Lo for 15 confirmed oxygen-rich objects. They are comparable with AGB stars and fainter than real supergiants. Note the luminosities were derived based on the kinematic distances.

3 . CO lines were detected from 15 objects and show profile with a width of $20-40 \mathrm{~km} / \mathrm{sec}$. This is the typical of evolved stars(but IRAS16279-4759 shows narrow profile from molecular cloud). In all $16 \mathrm{OH}$ maser sources the $1612 \mathrm{MHz}$ transition is dominate. This is also the typical of evolved stars with dense circumstellar envelope.

4. The scale height of sample is $\mathrm{Z}_{0}=0.27 \mathrm{kpc}$ (based on the kinematic distances) which is comparable with planetary nebulae of $0.3 \mathrm{kpc}$.

5 . We have checked the optical variation of 24 southern objects which are with optical counterparts, all are nonvariable(but IRAS14122-5947).

Hu J.Y., de Jong T., Slijkhuis S.,1990, 'From Miras to Planetary Nebulae', 487-489. 